Nephrologe 2021 · 16:203

https://doi.org/10.1007/s11560-021-00508-z

Angenommen: 16. April 2021

(c) Springer Medizin Verlag $\mathrm{GmbH}$, ein Teil von Springer Nature 2021

Schon lange wurde der Ernährung hinsichtlich Entstehung und Progression chronischer Nierenerkrankungen („chronic kidney disease“, CKD) eine große Bedeutung zugemessen. Zunächst standen die Progressionshemmung und das Hinauszögern der Dialysepflicht im Vordergrund. In den letzten Jahren kam als mindestens gleichbedeutendes Ziel die Reduktion des kardiovaskulären Risikos hinzu. Bei der Progressionshemmung lag der Fokus fast ausschließlich auf einer diätetischen Eiweißrestriktion, andere Nahrungsbestandteile wurden lange als weniger relevant betrachtet. Zunehmend wird jedoch klarer, dass auch andere Aspekte der Ernährung, wie die Beeinflussung von Stoffwechselwegen, der Einfluss auf das Mikrobiom des Gastrointestinaltrakts oder eine durch übermäßige Kalorienzufuhr ausgelöste Adipositas eine wichtige Rolle in der Pathogenese und im Verlauf von Nierenerkrankungen spielen können. Aktuelle Entwicklungen aus diesen neuen Forschungsbereichen sollen im Mittelpunkt dieser Ausgabe stehen.

Die Abnahme der glomerulären Filtrationsrate (GFR) im Verlauf von CKD wird auch durch den natürlichen Alterungsprozess beeinflusst. In einem sehr schönen Beitrag von Mafra et al. werden der Einfluss verschiedener Nahrungsbestandteile auf zelluläre Alterungsprozesse bei CKD und sich hieraus ergebende therapeutische Optionen dargestellt. So können spezifische Diäten bzw. Nahrungsbestandteile wichtige pathophysiologische Prozesse der Progression durch Beeinflussung von Entzündungs- und Alterungsprozessen vermindern.

Eine besondere Bedeutung für die Entwicklung einer CKD hat wohl auch das intestinale Mikrobiom, also die Ge-

\author{
Jens Lutz ${ }^{1}$ Martin K. Kuhlmann ${ }^{2}$ \\ ${ }^{1}$ Klinik für Innere Medizin - Nephrologie, Infektiologie, Gemeinschaftsklinikum Mittelrhein, Standort \\ Kemperhof, Koblenz, Deutschland \\ ${ }^{2}$ Klinik für Innere Medizin - Nephrologie, Vivantes Klinikum im Friedrichshain, Berlin, Deutschland
}

\title{
Niere und Ernährung
}

samtheit der Darmbakterien und ihre individuelle Zusammensetzung. In einem sehr aktuellen Beitrag von Fleig wird anschaulich dargestellt, dass es durch Fehlbesiedlung des Darms im Sinne einer sog. Dysbiose zu einer vermehrten Bildung toxischer Metaboliten kommen kann, die bei Nierenfunktionsverminderung akkumulieren. Dadurch kann nicht nur die Progression der CKD negativ beeinflusst, sondern auch das Auftreten kardiovaskulärer Komplikationen gefördert werden. Hier handelt es sich um ein sehr spannendes Forschungsgebiet mit möglicherweise sehr relevanten Implikationen für das Wohl unserer CKD-Patienten.

Auch bei der schweren Adipositas durch unverhältnismäßig hohe Kalorienzufuhr spricht man seit Langem von einer Epidemie. Die Adipositas kann sowohl durch mechanische, aber auch durch metabolische Faktoren, wie die Förderung eines entzündlichen Milieus, die Entstehung und das Fortschreiten einer CKD beeinflussen. In einer sehr klaren Übersichtsarbeit stellen Pommer und Zorron die möglichen therapeutischen Optionen zur Behandlung einer Adipositas bei Patienten mit CKD dar und zeigen, wie dies den Krankheitsverlauf positiv beeinflussen kann.

Bekanntermaßen spielen Autoimmunkrankheiten eine wichtige Rolle bei der Entwicklung akuter und chronischer Nierenerkrankungen. Dass sowohl die Entstehung als auch der Verlauf dieser Nierenerkrankungen durch die Ernährung beeinflusst werden können, haben Boedecker und Weinmann-Menke in eigenen Forschungsarbeiten mehrfach nachweisen können. In ihrem Beitrag stellen die Autorinnen dar, durch welche Nahrungsstoffe z. B. eine Lupusnephritis in ihrer Entwicklung begünstigt wird und wie der Verlauf von Nierenkrankheiten mit autoimmuner Genese durch die Ernährung potenziell beeinflusst werden kann.

Insgesamt stellt diese Ausgabe aktuelle Erkenntnisse zum Zusammenhang zwischen Ernährung und CKD dar und gibt einen Ausblick auf zukünftige neue therapeutische Möglichkeiten, die dieses spannende und sich schnell entwickelnde Feld für unsere Patientinnen und Patienten mit CKD bereithält.

\section{Korrespondenzadresse

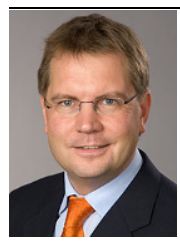 \\ Prof. Dr. Jens Lutz \\ Klinik für Innere Medizin - Nephrologie, Infektiologie, Gemeinschaftsklinikum Mittelrhein, Standort Kemperhof Koblenzer Str. 115-155, 56073 Koblenz, Deutschland Jens.Lutz@gk.de

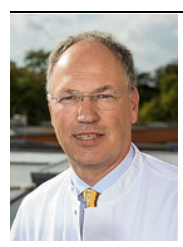 \\ Prof. Dr. \\ Martin K. Kuhlmann \\ Klinik für Innere Medizin - Nephrologie, Vivantes Klinikum im Friedrichshain Landsberger Allee 49, 10249 Berlin, Deutschland Martin.Kuhlmann@vivantes.de}

Interessenkonflikt. J. Lutz und M.K. Kuhlmann geben an, dass kein Interessenkonflikt besteht. 